\title{
Wellness Prediction Model for Hellenic Seamen Using Artificial Intelligence
}

\author{
A. Karagounis \\ Dept. of Industrial Design \\ and Production \\ Engineering University of \\ West Attica \\ Athens, Greece
}

\author{
N. Nikitakos \\ Dpt. of Shipping trade \& \\ Transport University of \\ Aegean, \\ Athens, Greece
}

\author{
D. Papachristos \\ Dept. of Industrial Design \\ and Production \\ Engineering University of \\ West Attica \\ Athens, Greece
}

\author{
M. Papoutsidakis \\ Dept. of Industrial Design \\ and Production \\ Engineering \\ University of West Attica, \\ Athens, Greece
}

\begin{abstract}
This paper presents a model of personal wellness prediction of Hellenic seafarers, based on mechanical learning with classification using the Exhaustive CHAID, ID3 algorithms and neural networks. The research is asked to answer the following research questions: "Is there a possibility of creating a model of personal wellness prediction through supervised mechanical learning? To what extent is this model acceptable and reliable? "And" can the binary classification with maximum information gain be applied? ". Training data was drawn from 900 samples of Hellenic naval engineers and captains completing training at the Navy Training Center (KESEN). The results of the research are that using Exhaustive CHAID with split-validation, Exhaustive CHAID with Crossvalidation, ID3 in Matlab environment and MLP with neural network methods, it is possible to create such a prediction model in which the sleep issues parameter is the determining factor for the existence or not of personal wellness.
\end{abstract}

\section{Keywords}

Wellness, stress, Hellenic seamen, machine learning, neural networks, artificial intelligence.

\section{INTRODUCTION}

The International Committee of Seafarers' Welfare, recognizing the important role that lifestyle plays in health (mental and physical), promotes "MENTAL CARE" as one of the issues of the Seafarers' Health Information Program. At the same time, Framework Directive 89/391 / EEC, supported by framework agreements between the social partners on work-related stress and harassment and violence at work, is mentioned in Section II, Article 6, paragraph 3 (b) of the said Decision "Where an employer assigns duties to a worker, he shall take into account the health and safety of that worker". In February 2006, the International Maritime Labor Conference adopted the Maritime Labor Convention. This Convention lays down minimum requirements for the health, safety and wellness of seafarers, also described as the fourth pillar of maritime legislation, complementing the current legislation of STCW, SOLAS and MARPOL, which suggests that this convention is very much complex and extremely broad in its scope.

The personal wellness of a person is a determining factor in the existence or absence of anxiety. Through studies and extensive research especially in business settings, knowing whether an employee feels good and wellness is an important factor for the employer and the employee in his / her career as well as for making decisions under unfavorable conditions. It is an important factor because many accidents can be avoided by preventing the consequences of working for an anxious person without having the right thinking and controlling association.

The research questions are the following:

- Is it possible to create a model of personal wellness prediction through supervised mechanical learning? To what extent is this model acceptable and reliable?

In order to better approach the research question, the following research sub-question is answered below:

- Can the binary classification with the maximum gain of information be applied?

\section{WELLNESS AND ARTIFICIAL INTELLIGENCE}

According to the World Health Organization, "mental health" is defined as "a state of wellness, in which each person realizes his or her potential, can manage the stress of everyday life, works efficiently and fruitfully and has the potential to contribute to his community". In short, to be mentally healthy, you must be physically, mentally and socially healthy. (Mental Health of Seafarers \& Disorders 2016).

According to Selye (1956), stress could be described as the common denominator of all the adaptive responses of the organism, or as the non-specific response of the organism to any requirement whether caused by or resulting in pleasant or unpleasant situations. However, the term usually refers to a situation that always exists in a person, which is though intensified when there is a change or threat that the individual has to deal with. Many studies have linked stress to physical, mental and social health, starting with Cannon and Seyle's research.

In the research by Carotenuto et al. (2012), it was confirmed that shipping is associated with mental, psychosocial and physical anxiety factors. The most important factors were family separation, loneliness on board, fatigue, multinationality, limited recreational activity, and sleep deprivation. The AMSA report provided a more detailed analysis of the lifestyle and the relevant factors that cause psychological discomfort. The repressors affecting the seafarers working in the engine room were different from those concerning the deck crew.

Additionally, the research by Jepsen et al. (2015) has shown that work at sea involves multiple risk factors for fatigue that, in addition to acute effects (e.g. reduced cognition, accidents), contribute through autonomous, immunological and metabolic pathways to the development of chronic diseases to seafarers.

Artificial Intelligence is the field of computer science, which 
deals with the design of intelligent computing systems, i.e. systems that exhibit characteristics related to intelligence in human behavior (Barr and Feigenbaum, 1981).

Mechanical Learning is defined as 'the creation of models or patterns from a data set, from a computational system' (Vlachava et al.,). Witten \& Frank (2000) define it as "something he learns when he changes his behavior in such a way as to perform better in the future".

The types of mechanical learning are as follows:

- $\quad$ Supervised learning

- Unsupervised learning

- $\quad$ Reinforcement Learning

\subsection{Artificial Neural Networks}

Artificial Neural Networks (ANN) are technological approaches to biological counterparts, on which the superior functions of beings are based, so they vary in application. They grow on the basis of organic networks and improve as the sample of input and output data with which they are 'trained' increases. The networks 'learn' and their operating mechanisms essentially incorporate the experience that is offered through these data (Anderson and Rosenfeld, 1988).

ANN process information and respond dynamically to external stimuli (inputs). Each artificial neuron consists of multiple inputs $x_{i}$ and a single $y$ output. Each input $x_{i}$ "weighs" with a weight $\mathrm{w}_{\mathrm{i}}$ and the results are summed through the summation function $\mathrm{F}$ :

$$
F=\sum_{i}^{n} x_{i} w_{i}
$$

The artificial neuron outputs through the transfer function only when the weighted sum of the inputs is greater than a certain threshold value $\theta$, i.e. when:

$$
\sum_{i}^{n} x_{i} w_{i}-\theta>0
$$

An artificial neuron is a simplified model of the natural neuron in that interconnecting weights form the electrical characteristics of the junction contact and the threshold value simulates the saturation behavior of the natural neuron.

\subsection{Decision Trees}

Decision Trees (DT) is the most well-known Induced Learning algorithm and has been successfully applied in many areas where classification is required: for example, in the identification of faces in images, in medicine for incident diagnosis, in predictions necessary for advertising, products and, more generally, for knowledge mining. The DT algorithm leads to the creation of a tree-like form whose sheets are classes. This tree form can be read as a set of rules called classification rules and gives a convincing answer to the question: "How can a machine create general rules from specific observations and how credible are these rules in action?".

\section{METHODOLOGY}

The training data was drawn from a sample of Greek naval engineers and captains completing training at the Navy Training Center (KESEN), with the relevant written permission from the relevant Ministry of Merchant Marine and Island Policy. The number of the study sample collected is 900 samples $(7.1 \%$ of all Greek seamen, according to the census made in 2014, where the number of seafarers working on Greek and Greek-owned ships was 22,925, of which 12.663 with Greek citizenship).

The "black box" model was used to study the learning model and export the results. Types of mechanical learning have been chosen as supervised learning.

In this case, the system has to "learn", i.e. to construct a new model in the form of a predictor function, which will represent given inputs to known, desired outputs, with the ultimate goal of generalizing this function and for inputs with an unknown exit. For the forecasting function, the following apply:

Each input, whether given or not, which the function can accept is characterized as an instance, thus creating a set of instances. Inputs are described based on the attributes they possess and have been characterized as significant since the beginning of the study of the problem that the system is called to solve. The given inputs are compiled by observations and form the so-called training set, which is a subset of the set of instances. The rest of the set of instances is the test set to be used during the certification phase. The function depicting an input from the training set to its known output is called a goal function. The value returned by the target function for an instance of the set of instances is given to a variable called goal variable. In supervised learning, the behavior of the target function is improved through learning processes with the help of the error function that detects the difference of the target variable from the desired output.

In the present thesis, 900 instances were drawn from research on the effects of the naval profession on the psychology of Greek seafarers (ELINT, 2017). These instances, each of which is a Greek Navy employee, have attributes that are related to their personal wellness and in particular are the following:

Instances attributes:

- Have there been times when you missed your thoughts when you had to do something fast?

- Did you have difficulty falling asleep or waking up in the evening?

- Have you been in a state of great tension or nervousness?

- Has committing suicide occurred to you?

- Did you often feel tired, exhausted, without energy?

The above features have five-stage values with the following meanings and were considered in the present study as Nominal type:

\section{Not at all 2 Very little 3 Moderate 4 Enough 5 Too much}

For each given education with the values of their attributes, it was decided to categorize it for YES and non-personal wellness (NO). The label, therefore called EUEXIA, is the distinct categorization of training data. The selection criterion for a person with a personal wellness (YES) is to have an average value of less than 2.5 , or an average of over 2.5 leads to unhealthy individuals. The decision boundary was done without decision cost analysis following the common sense of half of the five-tier scale. 
For the implementation of the mechanical forecasting model was chosen:

i. Classification by decision tree. The SPSS statistical program was used to make the decision tree finding analysis using the CHAID method and the MATLAB mathematical program using the ID3 algorithm.

ii. Learning neural networks using the SPSS statistical program by doing MLP (Multilayer Perceptron)

The criterion of comparing these methods is the best possible creation of a decision tree with the greatest information gain and hence the smaller entropy.

\section{EXPERIMENTAL RESULTS}

Instances classification: Using the IBM SPSS (v.20) statistical program, we define the instance attributes with the absentmindedness, sleeplessness, anxiety, suicide, exhaustion as Nominal type input data as the target for the euexia variable.

\subsection{Chi-squared Automatic Interaction Detection method}

For the development and creation of the decision tree, in order to sort the instances, the CHAID method was used. Euexia was defined as a dependent variable and the other traits as independent variables (predictors). At each step, the method selects the predictor that has the greatest effect on the dependent variable. Categories of each independent variable are merged if they do not have a significant difference with respect to the dependent variable. The Exhaustive CHAID method examines all possible separations of each independent variable.The validation process allows us to evaluate how well the structure of the trees generates for a large number of data. There are two ways to validate data:

\subsubsection{Exhaustive CHAID method with split-validation} In this case, the tree model is created using training data and control data. It was selected from a total of 900 screenshots of $75 \%$ as training data (675) and $25 \%$ of test data (225). Fig. 1 shows the structure of classification tree for data training. The value $\mathrm{NO}$ of the dependent variable euexia presents with blue color and the value YES presents with green color.
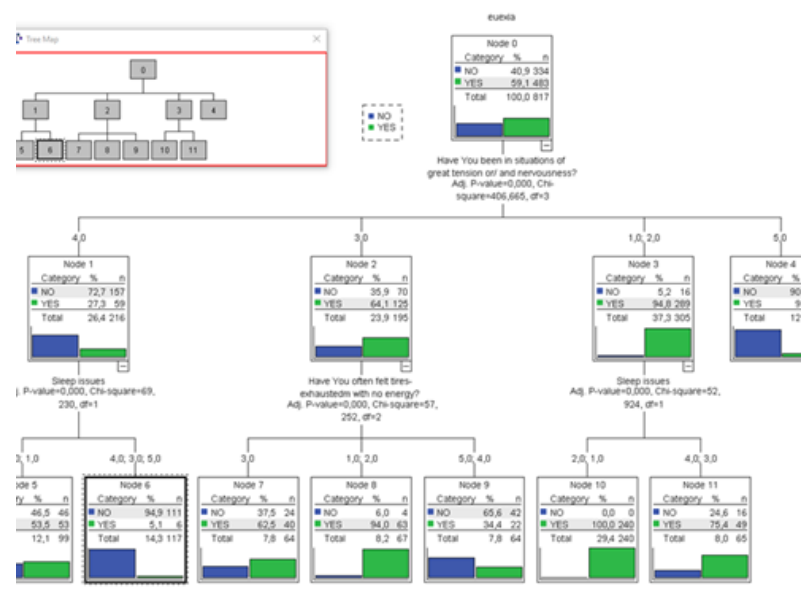

Fig 1: Data training structure tree

Figure 2 shows the structure of classification tree for data test.
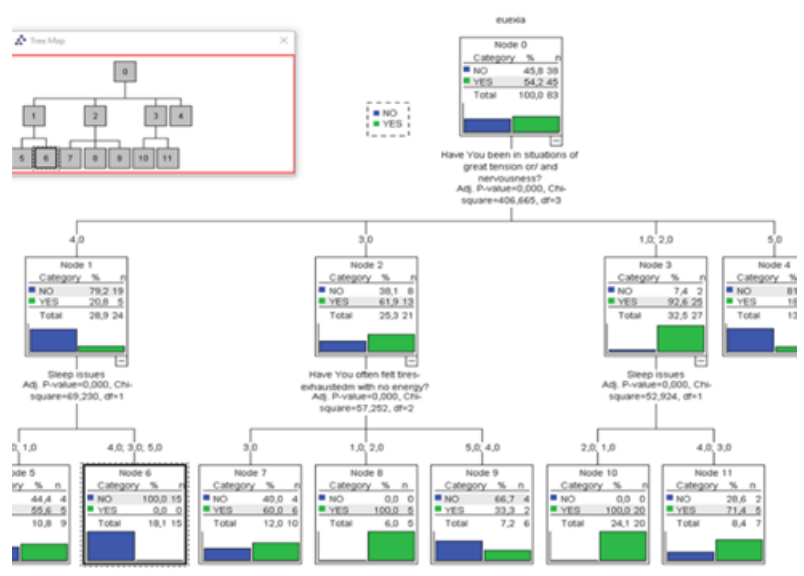

Fig 2: Data test tree structure

Table 1 shows the risk analysis of the specific methodology, which is a measure of the foreseeability of the tree. For training data the error rate is $15.7 \%$ and for the control data $16.9 \%$.

Table 1. Risk estimation

\begin{tabular}{|c|c|c|}
\hline Sample & Estimate & Std. Error \\
\hline Training &, 157 &, 013 \\
\hline Test &, 169 &, 041 \\
\hline \multicolumn{2}{|c|}{ Growing Method: EXHAUSTIVE } \\
CHAID Dependent Variable: euexia \\
\hline
\end{tabular}

Table 2 shows the results of the classification. This table shows the number of cases correctly and incorrectly classified for each dependent variable category with a total prediction rate of $84.3 \%$ for training data and $83.1 \%$ for test data.

Table 2. Classification with split-validation

\begin{tabular}{|c|c|c|c|c|}
\hline Sample & Observed & \multicolumn{3}{|c|}{ Predicted } \\
\cline { 3 - 5 } & & NO & YES & $\begin{array}{c}\text { Percent } \\
\text { Correct }\end{array}$ \\
\hline \multirow{4}{*}{ Training } & NO & 244 & 90 & $73,1 \%$ \\
\cline { 2 - 5 } & YES & 38 & 445 & $92,1 \%$ \\
\cline { 2 - 5 } & Overall Percentage & $34,5 \%$ & $65,5 \%$ & $84,3 \%$ \\
\hline \multirow{3}{*}{ Test } & NO & 28 & 10 & $73,7 \%$ \\
\cline { 2 - 5 } & YES & 4 & 41 & $91,1 \%$ \\
\cline { 2 - 5 } & Overall Percentage & $38,6 \%$ & $61,4 \%$ & $83,1 \%$ \\
\hline \multirow{2}{*}{ Growing Method: EXHAUSTIVE CHAID Dependent Variable: } \\
\cline { 2 - 5 } euexia
\end{tabular}

\subsubsection{Exhaustive CHAID method with}

\section{Crossvalidation}

In this case, the sample is divided into a number of patterns or folds. Tree models are then produced, excluding data from each pattern in turn. The first tree is based on all cases except those of the first sample, the second tree is based on all cases except those of the second sample and so on. For each tree, the risk of incorrect classification is calculated by applying the tree to the pattern that is excluded for its creation. The number of slices of the sample was set to the maximum of 25 . 
Cross-validation produces a single final tree model. The risk assessment with this method for the final tree is calculated as the average risk for all trees. Fig. 3 shows the structure of classification tree for all samples. The value $\mathrm{NO}$ of the dependent variable euexia is presented with blue color and the value YES is presented with green color.

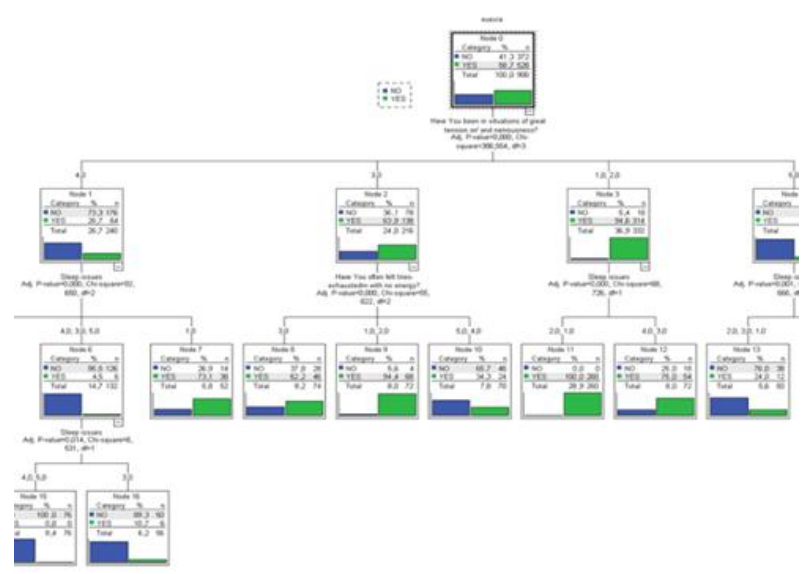

Fig 3: Samples tree structure

Table 3 shows the results of the classification. The overall predictive percentage of all data is $86 \%$ and therefore a $14 \%$ error rate.

Table 3. Classification with Crossvalidation

\begin{tabular}{|c|c|c|c|}
\hline \multirow{2}{*}{ Observed } & \multicolumn{3}{|c|}{ Predicted } \\
\cline { 2 - 4 } & NO & YES & Percent Correct \\
\hline NO & 308 & 64 & $82,8 \%$ \\
\hline YES & 62 & 466 & $88,3 \%$ \\
\hline $\begin{array}{c}\text { Overall } \\
\text { Percentage }\end{array}$ & $41,1 \%$ & $58,9 \%$ & $86,0 \%$ \\
\hline $\begin{array}{c}\text { Growing Method: EXHAUSTIVE CHAID Dependent Variable: } \\
\text { euexia }\end{array}$ \\
\hline
\end{tabular}

\subsection{ID3 algorithm method in Matlab environment}

Using mathematical Matlab (version R2016b), an appropriate tree classification code was developed based on the ID3 algorithm. The variable euexia is the class of the attributes of the absentmindedness, sleeplessness, anxiety, suicide, exhaustion. The data is imported from an external file in which values have been normalized to 0 and 1 values. The value 0 corresponds to a false state while the value 1 is true. The syntax of classification function: function [] = decisiontree (inputFileName, trainingSetSize, numberOfTrials, verbose) with the appropriate arguments leads to the result of ID3 sorting data. The inputFileName is the argument of the function in which we give the path of the data file. TrainingSetSize is the argument that determines the size of the training set of data. NumberOfTrials is the argument that determines how many times a decision tree will be created by randomly selecting a subset of data. Verbose is the argument that specifies the format of the tree results, 0 for a concise description 1 for a detailed description. Fig. 4 shows the results of classification using 10000 number of trials, 750 training set size for each trial and 150 testing set size, with a percentage of decision tree over all trials at $91 \%$.

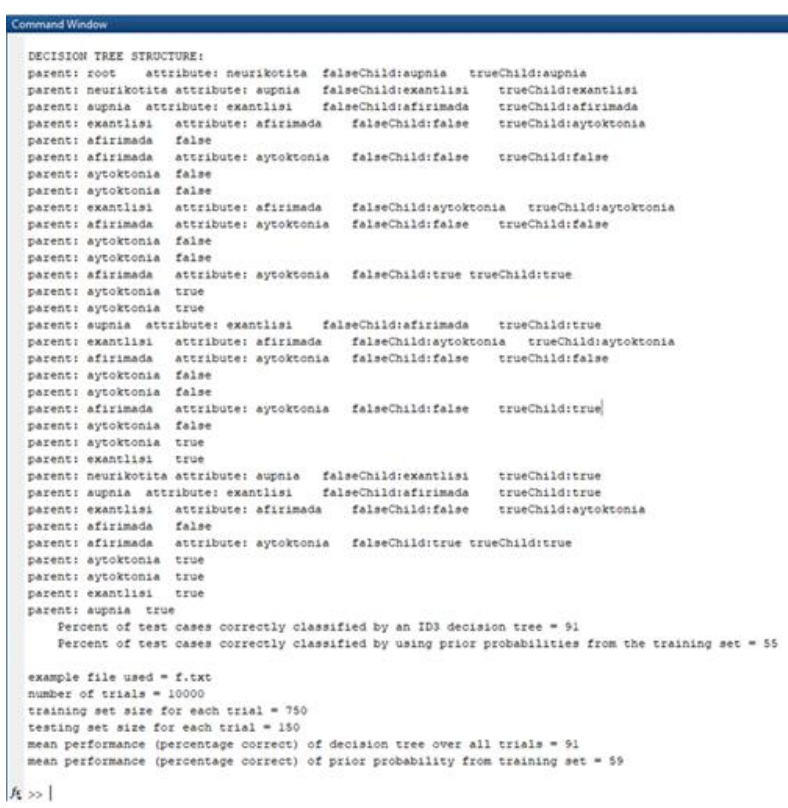

Fig 4: Classification with ID3 algorithm in Matlab

\subsection{MLP method with neural network}

Using IBM's SPSS (v.20) statistical program, the neural network MLP was selected to sort the data. The Perceptron (MLP) multilayer process produces a prediction model for one or more dependent (target) variables based on predictive variable values (independent variables). As a dependent variable, the welfare of the sailors (euexia) was defined and the variables absentmindedness, sleeplessness, anxiety, suicide, exhaustion, were defined as factors. The neural network architecture was set to two (2) hidden levels whereas the functions that connect the hidden layers and their units to each other are the sum of the weights of the inputs to the dependent variable. The function in hidden levels is excessive of the form $\gamma(\mathrm{c})=\tanh (\mathrm{c})$ while the function at the output level of the form $\gamma(\mathrm{c})=\mathrm{c}$. Of the total of 900 data, 631 samples $(70.1 \%)$ were obtained as training data and 269 (29.9\%) as randomized control data as shown in Table 4.

Table 4. Case processing summary MLP method

\begin{tabular}{|c|c|c|c|}
\hline \multicolumn{4}{|c|}{ Case Processing Summary } \\
\hline \multirow{2}{*}{ Sample } & Training & 631 & $70,1 \%$ \\
\cline { 2 - 4 } & Testing & 269 & $29,9 \%$ \\
\hline \multicolumn{2}{|c|}{ Valid } & 900 & $100,0 \%$ \\
\hline \multicolumn{2}{|c|}{ Excluded } & 0 & \\
\hline \multicolumn{2}{|c|}{ Total } & 900 & \\
\hline
\end{tabular}

The type of training that determines how the network processes the training data records was defined as a Batch. This technique only updates the summary weights after passing all the records of the training data, ie it uses information from all the files in the training data set, minimizing the total error directly by lamda, sigma, center interval, offset interval. Fig. 5 illustrates the structure of the neural network that classifies the data where the gray-colored lines have a positive weight, while those with a blue have a 
negative synaptic weight.

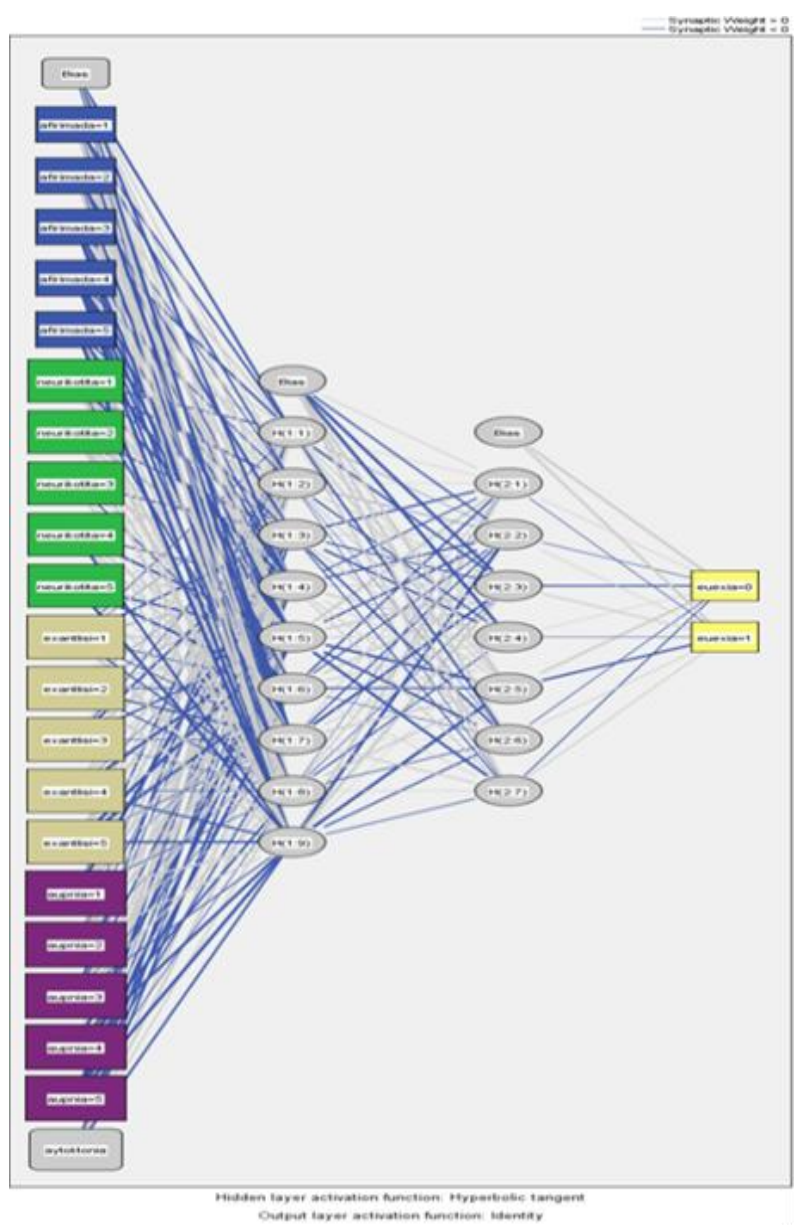

Fig 5: Neutral network structure

Table 5 presents the results of the classification of training and test data with a neural network separately for the two categories of wellness NO, YES and the overall prediction rate of $100 \%$.

Table 5. Prediction percentage for training and test data

\begin{tabular}{|c|c|c|c|c|}
\hline \multicolumn{3}{|c|}{ Classification } \\
\hline Sample & \multirow{3}{*}{ Observed } & \multicolumn{3}{c|}{ Predicted } \\
\cline { 3 - 5 } & & NO & YES & Percent Correct \\
\hline \multirow{4}{*}{ Training } & NO & 251 & 0 & $100,0 \%$ \\
\cline { 2 - 5 } & YES & 0 & 380 & $100,0 \%$ \\
\cline { 2 - 5 } & $\begin{array}{c}\text { Overall } \\
\text { Percent }\end{array}$ & $39,8 \%$ & $60,2 \%$ & $100,0 \%$ \\
\hline \multirow{4}{*}{ Testing } & NO & 121 & 0 & $100,0 \%$ \\
\cline { 2 - 5 } & YES & 0 & 148 & $100,0 \%$ \\
\cline { 2 - 5 } & $\begin{array}{l}\text { Overall } \\
\text { Percent }\end{array}$ & $45,0 \%$ & $55,0 \%$ & $100,0 \%$ \\
\hline \multicolumn{5}{|c|}{ Dependent Variable: euexia } \\
\hline
\end{tabular}

Table 6 shows the significance of the independent variables in relation to the dependent variable. Sleep issues are most important with $22.4 \%$ and $100 \%$ normalization rates.
Table 6. Independent variable importance

\begin{tabular}{|c|c|c|}
\hline Independent Variable Importance & Importance & $\begin{array}{c}\text { Normalized } \\
\text { Importance }\end{array}$ \\
\hline $\begin{array}{c}\text { Have there been times You lost focus } \\
\text { during a pressing situation? }\end{array}$ &, 155 & $68,9 \%$ \\
\hline $\begin{array}{c}\text { Have You been in situations of great } \\
\text { tension or/ and nervousness? }\end{array}$ &, 208 & $92,7 \%$ \\
\hline $\begin{array}{c}\text { Have You often felt tires-exhausted } \\
\text { with no energy? }\end{array}$ &, 214 & $95,2 \%$ \\
\hline Sleep issues &, 224 & $100,0 \%$ \\
\hline Have You considered suicide? &, 199 & $88,7 \%$ \\
\hline
\end{tabular}

Captions should be Times New Roman 9-point bold. They should be numbered (e.g., "Table 1" or "Figure 2"), please note that the word for Table and Figure are spelled out. Figure's captions should be centered beneath the image or picture, and Table captions should be centered above the table body.

\section{CONCLUSIONS}

In this work, we investigate a wellness prediction model for Hellenic seamen using different methods. All methods lead to the assumption that it is possible to predict the wellness of seamen with a forecast percentage above $80 \%$. A prediction model with neural network is better than any other method is. The initial research questions and sub questions are being validated through the results in section 4 . Table 7 shows the comparison between four methods.

Table 7. Comparison of prediction methods

\begin{tabular}{|c|c|c|}
\hline Method & $\begin{array}{c}\text { Prediction } \\
\text { rate of } \\
\text { training data }\end{array}$ & $\begin{array}{c}\text { Prediction rate } \\
\text { of test data }\end{array}$ \\
\hline $\begin{array}{c}\text { Exhaustive CHAID } \\
\text { with split-validation }\end{array}$ & $84.3 \%$ & $83.1 \%$ \\
\hline $\begin{array}{c}\text { Exhaustive CHAID } \\
\text { with Crossvalidation }\end{array}$ & \multicolumn{2}{|c|}{$86 \%$} \\
\hline $\begin{array}{c}\text { ID3 algorithm in } \\
\text { Matlab }\end{array}$ & $100 \%$ & $100 \%$ \\
\hline $\begin{array}{c}\text { MLP with neural } \\
\text { network }\end{array}$ & & \\
\hline
\end{tabular}

\section{ACKNOWLEDGMENTS}

All authors would like to thank the University of West Attica and specifically the Post Graduate Program of Studies (MSc) "New Technologies in Shipping and Transport", for the financial support provided to them to undertake this research project.. 


\section{REFERENCES}

[1] Simon, H.A (1981). The Sciences of Artificial. MIT Press

[2] Forsythe R. \& Rada R. (1986). Machine Learning: Applications in Expert Systems and Information Retrieval. E. Horwood

[3] Carbonell \& Langley, 1983. Machine Learning in S. Shaphiro: Encyclopedia of A.I., Wiley.

[4] Michalski, Carbonell and Mitchell 1983. Machine Learning: An Artificial Intelligence Approach, Vol 1, Tioga Publishing

[5] Nikolaou G.,Advanced Systems Control Topics, Teaching notes of the postgraduate curriculum, Piraeus 2017

[6] Diamantaras K.,Artificial Neural Networks, Kleidarithmos Publications, Athens 2007

[7] Goldberg, David E.; Holland, John H. (1988). «Genetic algorithms and machine learning». Machine Learning 3

[8] Michie, D.Spiegelhalter, D. J..Taylor, C. C. (1994). Machine Learning, Neural and Statistical Classification. Ellis Horwood.

[9] Mehryar Mohri, Afshin Rostamizadeh, Ameet Talwalkar (2012) Foundations of Machine Learning, MIT Press

[10] EthemAlpaydin "Introduction to Machine Learning The MIT Press, 2010

[11] Simon Haykin, "Neural networks and learning machines 3rd ed.”, Prentice Hall, 2009

[12] John Slavio, "Deep Learning and Artificial Intelligence: A Beginners' Guide to Neural Networks and Deep Learning”, Abhishek Kumar, 2018

[13] Stuart Russell, "Artificial Intelligence: A Modern Approach Paperback”, Pearson Prentice Hall, 2015.

[14] Ajay Agrawal, Joshua Gans, Avi Goldfarb, "Prediction Machines: The Simple Economics of Artificial Intelligence", Pearson Prentice Hall, 2018

[15] Pao,Yoh-Han, "Adaptive Pattern Recognition and Neural Networks", Wokingham, USA: Addison-Wesley, 1989.

[16] Russel, S. J. ,\& Norvig, P., "Artificial Intelligence-A Modern Approach ( $2 \eta$ edition)", Upper Saddle River, New Jersey: Pearson Prentice Hall, 2003.

[17] Vlaxavas I., Kefalas P., Vasiliadis N., Refanidis I., Kokkoras F. \& Sakellariou, "Artificial Intelligence (3rd Edition)", Thessaloniki: University of Macedonia Publishing, 2011.

[18] Mitchell, T.M., "Machine Learning”, H.B.:McGraw-Hill International Editions, 1997.

[19] Barr, A. and Feigenbaum, E. A. 1981. The Handbook of artificial intelligence, volume 1, Stanford, Calif.: Heuris Tech Press ; Los Altos, Calif. : William Kaufmann

[20] Vlachavas I., Notes to Artificial Intelligence. Introduction to Artificial Intelligence. Thessaloniki, 2013

[21] Forsythe R. \& Rada R. (1986). Machine Learning: Applications in Expert Systems and Information Retrieval. E. Horwood
[22] Michalski, Carbonell and Mitchell 1983. Machine Learning: An Artificial Intelligence Approach, Vol 1, Tioga Publishing

[23] Jepsen J., R., Zhao Z. and Leeuwen W., M.A, 2015. Seafarer fatigue: a review of risk factors, consequences for seafarers' health and safety and options for mitigation. IntMarit Health, 66, 2: 106-117, Via Medica.

[24] Witten, I.H., Frank, E. (2000), Data Mining: Practical Machine Learning Tools and Techniques with Java Implementations Morgan Kaufmann, San Mateo, CA, 2000 .

[25] Kambourlazos V. and Papakostas G., 2015. Introduction to Computational Intelligence. Greek Academic Electronic Signs and Assistive, ISBN: 978-960-603078-9

[26] Anderson, J. A. and Rosenfeld,E., 1988 Neurocomputing: Foundations of Research, MIT Press, Cambridge.

[27] Widrow, B., and Lehr, M. A, 1990. '’30 years of adaptive NN: Perceptron, Madaline and Backpropagation', Proc. Of the IEEE, vol. 23.

[28] Lippmann. R. P. 1987. 'An introduction to Computing with NN', IEEE ASSP Magazine, vol. 5

[29] Kohonen, T., 1984. Self - Organization and Associative Memory, Springer Verlag, Berlin.

[30] Selye H., 1956. The stress of life. New York, McGraw Hill Book Company (2nd ed, 1978)

[31] Byrne M.J., Thompson LF. Key concepts for the study and practice of nursing, 2nd ed. Saint Louis, CV Mosby Company.

[32] Dohrenwend, B. p., Shrout, P. E., Egri, G. and Mendelson, F. S. (1980). What pshychiatric screening scales measure in the general population: II. The components of demoralization by contrast with other dimensions of psychopathology. Archives of General Psychiatry, 37.

[33] Benson, H. (Ed.). (2000). Foreword: Twenty-fifth anniversary update. In The relaxation response (pp. 1 45). New York: Harper Torch

[34] Elo A. L., 1985. Health and stress of seafarers. Scandinavian Journal of Work, Environment \& Health $11,427-432$.

[35] Farber Barry A., (1983). Stress and Burnout in the Human Service Professions. New York: Pergamum Press.

[36] Moghaddum K.M. et al.(2013). Guidelines to Reducing Fatigue in Seafarers. Science explorer Publications.

[37] European Commission (2017). Seafarers: New measures to improve working conditions.

[38] Carotenuto A., Molino I., Fasanaro A., M. and Amenta F., 2012. Psychological stress in seafarers: a review. IntMarit Health 2012; 63, 4: 188-194, Via Medica

[39] Campbell, A., Converse, P., Rodgers, W. L. (1976). The quality of American life: Perceptions, evaluation and satisfactions. New York: Russell Sage Foundation. 